\title{
El libro como sistema: hacia un nuevo concepto de libro
}

\section{Julio Alonso Arévalo}

\section{José Antonio Cordón García}

\section{Recibido: 10 de septiembre de 2015}

\section{Aceptado: 22 de septiembre de 2015}

\section{Resumen}

El tiempo que vivimos no es precisamente una época de certezas. Nada de lo que ahora conocemos, ni siquiera el último descubrimiento científico, tiene garantizado un espacio en un planeta hiperconectado y en permanente evolución. En este contexto lo que fundamentalmente se está produciendo es un desplazamiento de lo analógico hacia lo digital. En este artículo analizamos la configuración de un nuevo concepto de libro que con su transformación digital está operando más allá del objeto para convertirse en un sistema de comunicación transmedia a través de tres procesos que son las tecnologías disruptivas, la desintermediación y la socialización del libro y la lectura.

\section{Palabras clave}

Libros electrónicos, lectura social, lectura digital, hipermedia, transmedia, multimedia, desintermediación, tecnologías disruptivas.

\section{The book as a system: toward a new concept of book}

\section{Abstract}

The times we live in are not exactly times of certainties. Nothing we now know, even the latest scientific discovery, is guaranteed a space in a hyperconnected and fast-changing planet. In this context, we are witnessing a shift from analog to digital. In this paper we discuss the development of a new concept of book which, with its digital transformation, is operating beyond the object to be a transmedia communication system through three processes: disruptive technologies, disintermediation, and socialization of the book and the act of reading. Keywords: ebooks, social reading, digital reading, hypermedia, transmedia, multimedia, disintermediation, disruptive technologies.

\section{Keywords}

ebooks, social reading, digital reading, hypermedia, transmedia, multimedia, disintermediation, disruptive technologies.

http://dx.doi.org/10.5209/rev CDMU.2015.v26.50628 


\section{INTRODUCCIÓN}

El libro siempre ha sido considerado como el medio utilizado por una población de autores y de lectores para satisfacer sus necesidades de comunicación escrita en la distancia y en el tiempo, necesidades que han sido satisfechas durante más de quinientos años por la estructura profesional de producción y distribución de los escritos. Las definiciones del libro se han dividido entre aquellos que lo consideran principalmente en su materialidad, y aquellos que lo consideran desde la perspectiva que toma en consideración su carácter como portador de un mensaje desde el punto de vista sociológico y semiótico. Explicar un texto exclusivamente por su forma material sería absurdo y se incurriría en un simplismo. La identificación del mismo nunca ha representado problema alguno desde el momento en que el concepto estaba asociado a un soporte, el papel, que le confería un carácter unitario y, en cierto modo, totalizador en el que continente y contenido formaban parte de un objeto en el que ambos conceptos eran indisociables. Pero la aparición de tecnologías de la información electrónica ha cambiado radicalmente este concepto de libro. De hecho cada vez es más difícil conciliar las denominaciones que van apareciendo asociadas a los nuevos soportes con la percepción canónica que se tenía de este soporte. Si acudimos al diccionario de la Real Academia de la Lengua nos encontramos con múltiples acepciones de libro, varias decenas, todas asociadas al libro en papel. Además de la definición convencional, se recogen múltiples variantes vinculadas con diferentes experiencias profesionales y particularidades de lectura: Libro Amarillo, azul,, blanco, borrador, copiador, de acuerdos, de asiento, de becerro, de caballerías, de cabecera, de caja, de coro, de cuentas ajustadas, de escolaridad, de estilo, de familia, de fondo, de horas, de inventarios, de las cuarenta hojas, de la vida, de lo salvado, de mano, de memoria, de misa, de música, de oro, de surtido, de texto, diario, entonatorio, maestro, mayor, moral, penador, procesionario, ritual, rojo, sagrado, sapiencial, talonario, verde. Sin embargo toda una serie de fenómenos han provocado una transformación radical de la concepción vinculada al libro (Cordón, Gómez, Alonso, 2013), suscitando la aparición de formas y géneros inexistentes previamente. Una consulta al Diccionario Digital de Nuevas Formas de Lectura y Escritura, DINLE (http://dinle.eusal.es/) da cuenta de ello. Libro homotético, libro enriquecido, libro pop-up, libro transmedia, crossmedia, libro aplicación, etc. constituyen algunos ejemplos de esta transformación en la que lo tecnológico se imbrica con las nuevas formas de creación, distribución y consumo.

La relación y los medios de conexión entre texto, contexto, paratexto e intertexto ha cambiado con el tiempo, al hilo que se ha ido desarrollando la tecnología, dando lugar a una imagen, una idea y un concepto de libro que necesariamente ha de ser diferente a la que se había manejado tradicionalmente. En la corta historia de 
los libros electrónicos los avances tecnológicos han permitido nuevas formas de relación y vinculación de los textos. El resultado es una serie de cambios en el formato y características de los mismos, y en la forma en que se presentan al usuario. Al igual que los cambios en los márgenes cambiaron las prácticas de lectura en los primeros años de la imprenta, las innovaciones técnicas transforman también la manera de leer y percibir los textos.

Cuando la prensa de tipos móviles creó la oportunidad de incrementar considerablemente la cantidad de textos disponibles, los impresores se esforzaron para hacer que los libros se parecieran lo máximo posible a los manuscritos. Fueron necesarias varias décadas para que la diferenciación fuera asumida como una nueva lógica laboral. Los libros electrónicos están experimentando un proceso similar. La migración del libro impreso al formato electrónico, manteniendo las convenciones de impresión, ha sido el camino emprendido por muchas editoriales, reproduciendo en el ámbito digital los parámetros preexistentes. Por lo tanto, la discusión sobre la materialidad del libro no puede centrarse en elementos desagregados como el papel, la tinta, la encuadernación o el contenido, en el caso del libro impreso, sino que ha de implicar también el conjunto de procesos desarrollados para la producción del libro. La modificación de los procesos de producción afecta de manera fundamental a las nociones culturales de autoría, lectores, y forma literaria. Por ejemplo, el elevado coste de los materiales necesarios para producir un libro impreso obligó a la publicación selectiva de acuerdo con unos criterios editoriales que devinieron en factores críticos del sistema de publicación: calidad de los contenidos, capacidad de venta del autor, circulación de la temática, etc. El coste del producto final determinó, en gran medida, el número de lectores, de ahí que no resulte baladí la hipótesis de si la reducción de los costes de producción, imputable al entorno digital, implicará una ampliación del número de lectores. La reducción de los costes de impresión, gracias a la introducción de la linotipia, generó la aparición de la prensa escrita periódica a gran escala y de nuevos géneros y nuevos lectores, con importantes consecuencias de carácter social y político en términos de influencia y penetración de las ideas. La publicación electrónica comenzó en gran medida gracias a sus ventajas de costes sobre la producción impresa. Muchas formas de ebooks son relativamente baratas de producir, ya que no requieren de una gran infraestructura de producción y edición. Una de las consecuencias es que lo que conocíamos como auto-publicación comienza a constituirse en norma. La publicación selectiva ya no es algo impuesto por factores tecnológicos o económicos. Son muchas las empresas y sistemas que favorecen la autopublicación, constituyendo esta otro flanco de investigación, tanto desde el punto de vista de la autoría como desde el punto de vista empresarial. Plataformas como Amazon, Barnes and Noble o Apple han creado programas para la publicación directa por parte de los autores, sin necesidad de intervención editorial. Estas circunstancias obligan a una evaluación de los supuestos culturales 
acerca de la autoría, los lectores, el género, la accesibilidad y facilidad de uso de los recursos puestos a disposición de todas estas instancias (incluyendo el control de la calidad y la censura).

\section{LAS NUEVAS CARACTERÍSTICAS DE LOS LIBROS}

Es difícil establecer una lista de características generalizadas del libro, porque el término se refiere a dos conceptos distintos. Por un lado, es un tipo empíricamente mensurable de objeto físico, por otro, un tipo poderoso y complejo de metáfora, con fuerte contenido simbólico. El problema es que, en el entorno digital, el término libro ha devenido en una metáfora demasiado simple para capturar todas las variables posibles de los contenidos digitales y de la información navegable, manipulable e intervenible en su seno. De cualquier modo, sigue siendo una metáfora de gran potencia, que concilia un ecosistema propio en permanente crecimiento al hilo de los continuos desarrollos tecnológicos. Bibliógrafos como McKenzie (2005) ya habían reflexionado sobre un fenómeno singular asociado al libro discutiendo como la percepción cultural del libro como una unidad de totalización de la producción entra en contradicción con la heterogeneidad que implica la red, en la que la textualidad, carece de toda la carga simbólica implícita en este, diluida como esta en formas cada vez más prolijas. Esta disfunción opera a nivel del inconsciente colectivo en la aceptación de las nuevas formas de producción y reproducción de textos. Cuando Abba y Bjarnason (2015) titulan su obra como "This is not a book", un ensayo sobre el entorno editorial y tecnológico de los libros electrónicos, están apelando a un discurso conceptual cuyos límites sobrepasan las definiciones canónicas de la terminología tradicional. Consideran los autores que mantener el termino libro para todas las formas complejas, hibridas, que están surgiendo no es mas que una vertiente del cierto platonismo reindicente, la idea de que ciertas formas pueden existir, o que de que sus características pueden cobrar sentido, fuera de su entorno original, que las ideas abstractas pueden existir de una forma pura, aisladas de su contexto.

En este sentido los desafíos surgen de la misma terminología con el término e-book, que no afecta únicamente a las reproducciones electrónicas de los libros impresos, sino también a todos los textos electrónicos en los que subyace la metáfora bibliográfica anteriormente mencionada, aunque las propuestas tecnológicas la alejen cada vez más del carácter cerrado y autosuficiente del libro convencional. Por lo tanto es preciso estudiar las características de los nuevos libros, no mediante la asignación de las funciones exclusivas de la textualidad digital, sino mediante la identificación de las características inherentes al sistema en el que circulan y están involucrados. Esto es:

Tangibilidad, o la capacidad de un libro para transmitir información sobre sí mismo a través de indicadores físicos tales como el tamaño y formato. Los libros electrónicos son considerados como intangibles, o tangibles de diferentes maneras, pero poseen unas características físicas que se deben considerar en su análisis. 
Características que oponen lo estático a lo dinámico, lo rígido a lo fluido, tal y como señalan Soccavo (2015) o Panoz (2013) que apelan al distanciamiento de la "página" como unidad de análisis textual, difuminada en componentes fluidos fruto de la necesaria neutralidad tecnológica de las obras. La proliferación de pantallas ha obligado a la inserción de los textos en un universo de adaptabilidades múltiples, en el seno del cual un lector ha de poder moverse sin solución de continuidad: de la web, a la Tablet, de esta a la pantalla del móvil, con sistemas de sincronización que permitan una actualización permanente en esta diáspora lectora. Benhamou (2014), articula toda una teoría acerca del "lector vagabundo", aquel que circula por los escritos y pantallas sin solución de continuidad.

Personalización. La historia de la edición da fe de un conjunto de convenciones tipográficas y espaciales que le han ido confiriendo a los textos estructura, coherencia, estabilidad y legibilidad. Un conjunto de convenciones que en todo momento han decidido los editores, directores de colección, directores literarios, coordinadores científicos, etc y que respondía a la necesidad de contar con un prototipo reproducible de manera idéntica en cada una de las tiradas de la obra. El lector se encontraba con un texto cuyo estilo, espaciado, márgenes, mancha, tipografía, apariencia, y sistemas de búsqueda (tablas e índices), se habían establecido previamente a su puesta en circulación. Las únicas posibilidades de intervención sobre el texto radicaban en la incorporación de anotaciones o en los subrayados que el lector podía incorporar dejando su impronta sobre el libro, dando con ello lugar a un género, los marginalia, de gran repercusión e importancia para la historia cultural y de las mentalidades (Burke, 2009)

Con los textos electrónicos el lector adquiere un protagonismo inexistente en épocas precedentes, al tiempo que el editor pierde una de sus señas de identidad fundamentales, su imagen de marca, plasmada en el diseño de colecciones, de interiores y en los paratextos. Frente al dictado preestablecido de tipos de letra, tamaño, etc, anteriormente mencionados, fruto de unos procesos de producción, realización y maquetación constitutivos de la planificación de colecciones y de obras, dotados de fuerte significación simbólica (Chartier, 2000) y corporativa, en el entorno digital el lector establecer un elenco de preferencias que modifican todos los supuestos relacionados con la presentación formal del texto, modificando el tamaño de la letra, el estilo el interlineado, la mancha, las justificaciones, etc (Cordón, 2014). No es que se oponga la voluntad del lector a la del editor, o que, como equivocadamente se suele interpretar, el editor quede al margen de un proceso en el que el lector toma decisiones antes imputables a aquel en exclusividad. En efecto, la posibilidad de que el lector pueda disponer de un elenco de opciones más o menos amplias para leer y trabajar con el texto, depende igualmente de las decisiones que la editorial haya tomado sobre el diseño digital de las obras, asi como de las aplicaciones susceptibles de ejecutar los contenidos, como Panoz (2013) pone de manifiesto. En 
este sentido, aunque el entorno digital favorece la personalización, y esta constituye un elemento indisociable del mismo, las posibilidades de aprovechamiento y optimización están en función del tipo de dispositivo, de la plataforma donde se encuentre la obra y de la aplicación de lectura utilizada.

No existe una antinomia editor-lector, sino un circuito digital en el que las decisiones y elecciones de ambos tienden a la concurrencia. Ello no es óbice para la imagen editorial tienda a debilitarse por la modificación de las lógicas de visibilización de los contenidos en el ecosistema electrónico. Naomí Klein (2011) había establecido la potencia de las marcas en el posicionamiento de las empresas, de tal manera que los efectos de dominación ejercidos por la imagen se inscriben en los modos de consumo de manera irreversible. En el ámbito editorial la capacidad de reconocimiento de la empresa ha estado durante décadas fuerte impregnada por unos diseños que permiten identificar fácilmente colecciones y editoriales, singularizando las opciones de compra y determinando, en cierto modo, las elecciones mediante las fidelizaciones simbólicas. Los elementos consustanciales a esta fijación tienen que ver con lo que Genette (2001) había incluido bajo las denominaciones de paratextos, peritextos y epitextos, un conjunto de discursos y elementos señaléticos que aportan información conceptual y simbólica sobre una obra, ejerciendo de auténticos "umbrales" de la misma: nombre del autor, título, dedicatorias, resumen de cuarta de cubierta, fajas, camisa, etc. representan un recordatorio permanente de la obra en el ámbito impreso, en la medida en que su activación visual es permanente e independiente del lector, que se enfrenta a ella cada vez que ve o aborda la obra. Estos elementos actúan como una suerte de hitos topográficos que facilitan la identificación y la búsqueda de una obra en particular, o de otras de la misma colección. Todos estos elementos, aunque parcialmente presentes en los libros electrónicos, lo están únicamente con carácter indiciario y de manera ocasional por cuanto el lector se enfrentará a una cubierta sólo la primera vez que abra una obra, en la mayoría de las ocasiones inserta en una vorágine de portadas conformando sus estanterías. De tal manera que uno de los componentes fuertemente asentados en la industria editorial, sancionados por la tradición y la academia, el relativo a la imagen, icónica y textual de las obras, se va debilitando en favor de las posibilidades de gestión del contenido, con lo que esto implica para el editor desde el punto de vista de las marcas.

La personalización se refiere no solo a la posibilidad de intervenir sobre el texto sino también al de acceso a un sistema adaptado de lecturas gracias a las recomendaciones ad hoc que, mediante diferentes sistemas, plataformas y editoriales hacen llegar al interesado. Se trata de una línea de trabajo cada vez más importante para el mundo editorial que ha contemplado como los sistemas de información canónicos, tales como reseñas, críticas, revistas especializadas, han ido perdiendo vigencia en beneficio de las redes sociales y otros sistemas de comunicación colaborativa (Cordón, Alonso, Gómez, 2013). La etnografía lectora se transforma al hilo de 
las propuestas que, con dispares niveles de precisión y basándose en perfiles de lectura diseñados mediante algoritmos de mayor o menor complejidad, a partir de los rastros que va dejando el lector, van conformando una experiencia completamente singularizada.

El carácter especular, mimético, del libro electrónico, muy significativo en los primeros lanzamientos de prototipos, ha ido desapareciendo para configurar una realidad distinta a la de su referente impreso, desprendiéndose de aquellos elementos característicos de ese entorno en beneficio de otros privativos del ecosistema digital

Navegabilidad, o la capacidad de proporcionar acceso a diferentes tipos de información, tanto interna como externa, a través de medios táctiles, o sistemas de circulación inteligentes. Hipervínculos, enlaces internos, referencias y notas convierten al libro electrónico en una obra abierta y circulable por los dominios de discursos que rompen con la linealidad y la estructura fija del impreso, introduciendo sistemas de navegación que modifican las estrategias de lectura predeterminadas. La hipertextualidad se erige en un rasgo característico de los contenidos digitales. Sin embargo, la mayoría de los trabajos sobre el hipertexto lo han considerado desde un punto de vista tecnológico, en términos de nodos, enlaces, lenguaje de marcas, etc. Pero la hipertextualidad tiene un impacto sustancial en los aspectos simbólicos de los textos, en la manera en que leemos, aunque pocas investigaciones han tratado de formalizar esto, sobre todo desde el punto de vista semántico. La navegabilidad introduce una función determinante en el entorno digital académico, la referencialidad, o grado de intertextualidad mediante enlaces (explícitos o implícitos), así como grado en que las partes de un texto pueden hacer referencia a otros textos. Tanto una como otra potencian otra de las prestaciones privativas del universo digital la buscabilidad, o la capacidad de un texto de encontrar ocurrencias de términos, principios y finales de capítulos, páginas marcadas, notas, etc.

Transmedialidad, o la naturaleza compuesta de libros en la que intervienen propuestas tecnológicas distintas, pero bien articuladas. La evolución de la tecnología permite observar los cambios directos en las funciones y características del libro. Por ejemplo, la llegada de las columnas y los márgenes para imprimir en la imprenta permitió los marginalia, que generaron las notas a pie de página o al final. La transmedialidad representa una de las características significativas del libro electrónico en tanto que permite la incorporación de todo tipo de medias en un solo discurso. No se trata de una mera transposición o yuxtaposición de propuestas narrativas sino de una integración de las mismas con significancias complementarias e irrenunciables para la comprensión del texto (Scolari, 2013). La transmedialidad se ha plasmado de manera elocuente en los denominados libros aplicación en los que el concepto de corpus editorial adquiere su sentido más pleno. 
Actualización. Si el libro electrónico es abierto por sus posibilidades de navegación e intertextualidad antes mencionadas, lo es también por sus posibilidades de actualización permanente, gracias a la cual se produce lo que Creuzet (2015) ha denominado como la metáfora del manuscrito, esto es una obra que permite modificaciones y revisiones permanentes como si de un original, todavía en fase de preparación, se tratase. En el ámbito científico esta particularidad permite disponer de las sucesivas versiones de una obra, con las respectivas revisiones que va haciendo el autor. Los conceptos de libro líquido o libro vivo no son ajenos a este fenómeno.

\section{EL LIBRO EN EL CIRCUITO BIBLIOGRÁFICO Y EDITORIAL}

La realidad es que, durante estos últimos años, el ecosistema del libro ha experimentado un cambio radical en todos sus elementos. La aparición de los libros electrónicos y las prestaciones asociadas a los mismos a través de las aplicaciones de eReader y tablet, blog, plataformas y sistemas de lectura social, etc., han puesto en cuestión las definiciones canónicas asociadas al libro tradicional, lo que a su vez está trastocando las pautas y hábitos de lectura en particular.

Se transforma el papel de las librerías como núcleos de acceso al libro impreso, el papel de las bibliotecas como sitios de salvaguarda y difusión del saber, los derechos asociados a la función de autor y su dimensión económica, con la crisis del copyright y de la propiedad intelectual, la crisis de los intermediarios obligados a reinventarse e idear nuevas estructuras que les permitan sobrevivir en el contexto digital, en el que se está articulando una nueva cultura resultante de una economía del intercambio, colaboración, de la reputación, de la interactividad y de la integración, y en el que se ha producido la fractura digital con un nuevo concepto de lector cada vez más acostumbrado al uso de la lectura electrónica. De este modo se producen algunos fenómenos asociados a la cultura de lo digital que identifican y avocan casi indefectiblemente a un nuevo concepto de libro, que a medio plazo cada vez se distanciara del referente y paradigma conocido -el libro impreso-y que se irá adecuando progresivamente a las propiedades, características y posibilidades que brinda el formato digital conformando de este modo dos manifestaciones de un mismo producto cada vez más diferenciadas. La inserción del libro en el circuito bibliográfico-editorial se conforma a través de tres fenómenos, estrechamente relacionados con las características definidas anteriormente:

1. La disrupción

2. La desintermediación

3. La socialización 


\section{La disrupción}

La inmaterialidad del formato abre innumerables posibilidades en todos los sentidos, y también importantes retos que debemos de enfrentar. Lo digital está impactando en la forma de crear contenidos, de comunicarlos, de leerlos y, en el ámbito científico, en las nuevas posibilidades de medir el impacto de la investigación, ya que el formato digital obedece a una nueva forma de pensar, pues pensar en digital implica nuevos paradigmas como son lo social, lo abierto y el remezcla, valores que estaban ausentes en el contexto analógico. Responde con ello a un modelo de tecnologías disruptivas, es decir tecnologías o innovaciones que conducen a la desaparición de productos o servicios que durante mucho tiempo han tenido una amplia vigencia, pero que en la actualidad utilizan preferiblemente una estrategia no sostenible frente a la nueva tecnología que tiende hacia una progresiva consolidación en el mercado. Stevev Sinofsky (Sinofsky et al. 2014), ex directivo de Microsft, explica los retos que frente al sistema establecido enfrenta un producto "disruptivo». Distingue cuatro fases:

\section{Disrupción}

En una primera fase se produce el reemplazo de alguna solución existente, ampliamente utilizada y que hasta el momento ha sido satisfactoria (El libro impreso), sin que la nueva tecnología (eBooks) sustituya completamente a la precedente, llegando a ser considerada algo poco importante, casi experimental y que no va a afectar al modelo de negocio existente. Esto es lo que ocurrió con la aparición de los dispositivos lectores y con la llegada de los sistemas de autopublicación durante los años 2007-2009.

\section{Evolución}

La segunda fase es la de evolución, que describe cómo después de la ruptura inicial, la industria comienza a relajarse y permite que los nuevos agentes desarrollen sus ideas. El agente tradicional continúa su trabajo con normalidad, aunque en cierto modo tolera la nueva tecnología y empieza a incorporar cambios en su propio negocio. Esto mismo ocurrió con el crecimiento inicial de los ebooks que despertó el interés de los editores, y muchos de ellos adoptaron algunos de los elementos clave de los libros electrónicos sin renovarse e innovar su negocio. Se correspondería con los años 2010-2013 cuando los editores incorporaron contenidos digitales, pero en el fondo consideraban que se trataba del mismo contenido, aunque en formato digital.

\section{Convergencia}

La fase 3. Convergencia, se produce cuando las partes perturbadoras y los agentes tradicionales empiezan a trabajar juntos, ya que, según Sinofsky, las tecnologías más antiguas evolucionan por una necesidad de estabilización. Hay una amplia aceptación de la nueva tecnología y los primeros la adoptan, lo que permite a la industria resolver la situación. Esto se correspondería según Cuddyen (Cuddyen 2015) con la situación actual 
en 2015, esta fase el peligro fundamental está en la complacencia de los editores tradicionales con la situación actual, ya que consideran que esta situación se puede revertir y dejan de planificar para el futuro.

\section{Reimaginacion}

la fase 4: Re-imaginación es, en opinión de Sinofsky la "última etapa de la tecnología de interrupción «... cuando una categoría o la tecnología se ha re-imaginado desde el principio». Alude con un ejemplo a lo que está sucediendo en otros sectores como por ejemplo en el del Taxi con respecto a la tecnología de Uber, una aplicación tecnológica disponible en más de 270 ciudades y 50 países de todo el mundo, que pone en contacto usuarios pasajeros y usuarios conductores con tan sólo apretar un botón, lo que ha provocado airadas protestas de los profesionales del taxi.

Según Gareth Cuddyen (op. cit) actualmente estaríamos en la fase 3 de la teoría de las tecnologías disruptivas. Esta situación el autor la compara con la de la música. Cuando en 2011 las ventas de música empezaban a recuperarse, la industria musical pensó que el gran tsunami que estaba transformando el sector había terminado, a pesar de que las ventas digitales representaban el $64 \%$ del total, lo que provoco que entre los diferentes agentes existiera el consenso de que el mercado se había estabilizado y estaba de vuelta a la normalidad. Pero en ese mismo año un sueco puso en marcha Spotify, que después de sólo cuatro años en el mercado cuenta en la actualidad con 15 millones de suscriptores y 60 millones de usuarios activos en todo el mundo. El modelo de negocio de Spotify ha perturbado claramente la industria de la música, con artistas que ahora buscan innovadoras formas de edición y promoción que les permita generar un nivel de ingresos aceptable. Para el mundo de la edición es importante tener en cuenta estas experiencias que, aunque obedecen a productos, modelos y contextos diferentes nos pueden proporcionar algunas pautas de utilidad y una experiencia enriquecedora. Por lo que la estrategia a seguir en este contexto es estar atento a las nuevas tendencias y estar predispuestos a experimentar con ellas.

Por todo ello podemos afirmar que la tecnología no es un elemento neutro en la manera en qu leemos, producimos información, la gestionamos, organizamos o difundimos. El libro digital no es una mera transposición de blancos sobre negros, tan característicos y propios del formato impreso, a una pantalla, si bien en estas primeras etapas de la transición de lo analógico a lo digital ha sido así, por el hecho de ser un referente absoluto al que respaldan medio millar de años de subsistencia. Pero en el nuevo contexto digital ya se están empezando a producir nuevas prácticas comunicativas, a través de nuevas estructuras y géneros discursivos que están caracterizados por la no linealidad y la interactividad. El libro como objeto material desaparece, cada vez se involucra más con el software y se convierte en software. No en vano una de sus manifestaciones más innovadoras y rupturistas, como se indicaba anteriormente, es la obra convertida en 
aplicación, en "app", el libro aplicación, muy utilizado para libros infantiles y manuscritos de bibliotecas, involucrando todo tipo de desarrollos y prestaciones multimedia, o transmedia, esto es el desarrollo de un contenido sobre varios medias diferenciados, lo cual le abre las puertas al mundo de cualquiera de los sistemas de comunicación, convirtiéndose en una interfaz compartida.

\section{La desintermediación}

Si tuviéramos que elegir una palabra que representara la última media década sin ninguna duda sería "desintermediación". Los conceptos de autoría, crítica y recepción, profundamente asentados en el medio impreso, están cambiando sus competencias y significados al hilo de fenómenos únicamente imputables al contexto digital. Los autores ahora disponen de herramientas para publicar de forma más rápida, más inteligente $y$, en muchos casos, más eficaz que las editoriales tradicionales. Como resultado se están desarrollando plataformas que proporcionan mayor visibilidad y presencia en el mercado que la edición tradicional. Ahora el problema para un autor no es saber si un editor le publicara la obra, sino saber cómo encontrar a sus lectores, lo que entraña el desarrollo de competencias complementarias al mero hecho de escribir. El escritor se convierte en promotor de sí mismo.

Se puede decir que autopublicación -"Self-Publishing" - es la publicación de cualquier libro o cualquier otro medio por parte del autor de la obra, sin la intervención de un tercero establecido o editor. El autor es responsable del control de todo el proceso, incluyendo el diseño (cubierta / interior), formatos, precio, distribución, marketing y relaciones públicas. Según José Afonso Furtado (Furtado 2013), la revitalización de la autopublicación se produce a partir de 2006, con el resurgir de los dispositivos de lectura, y ha producido un fenómeno de reconstrucción de la cadena de valor tradicional en la edición, que a su vez está provocando diversos fenómenos disruptivos. Se produce una pérdida de autoridad de los sistemas convencionales de valoración y referencia, con una importancia creciente de redes sociales, blog, wikis, como sistemas de valoración (Cordón-García and Alonso-Arévalo 2011). Hasta el punto que los textos electrónicos cuestionan varias categorías muy arraigadas en nuestra cultura escrita y documental. De manera que el modelo digital produce un efecto de desintermediación en el cual solo los dos elementos extremos de la cadena de valor del libro son imprescindibles: el autor y el lector. Existen otra serie de miembros que pueden estar presentes o no, como son el agente editorial y el editor; pero que no son necesariamente imprescindibles; mientras otros de ellos, como el distribuidor y el librero, son perfectamente prescindibles, ya que la distribución se realiza en línea desde las plataformas editoriales. La autopublicación también es uno de los segmentos de mayor crecimiento editorial. Los libros electrónicos autopublicados continúan ganando terreno a los formatos impresos, ya que actualmente son el 45 por ciento de los ISBN que se asignaron en 2013. 
El vaciado rápido de intermediarios -ya sean editores, minoristas tradicionales, agentes medios del libro, o agentes de la cadena de valor de la publicación- es un fenómeno bien documentado, y animado por la llegada de las "intermediaciones delgadas," término que utiliza a menudo Glenn Fleishman, para referirse a las plataformas tecnológicas que permiten la producción directa, sin la intervención de la distribución y los esfuerzos de marketing que tradicionalmente han estado en manos de las grandes empresas. La adaptación de los diferentes agentes de mediación a este contexto no responde a una solución única, sino una variedad de ajustes contextuales y cambios de estrategia personalizados que permitirán a los agentes y empresas, cualquiera que sea su tamaño, seguir haciendo el trabajo que permite descubrir el talento, pulir, mejorar la obra, y llevar los libros al mercado (Ashlock 2014).

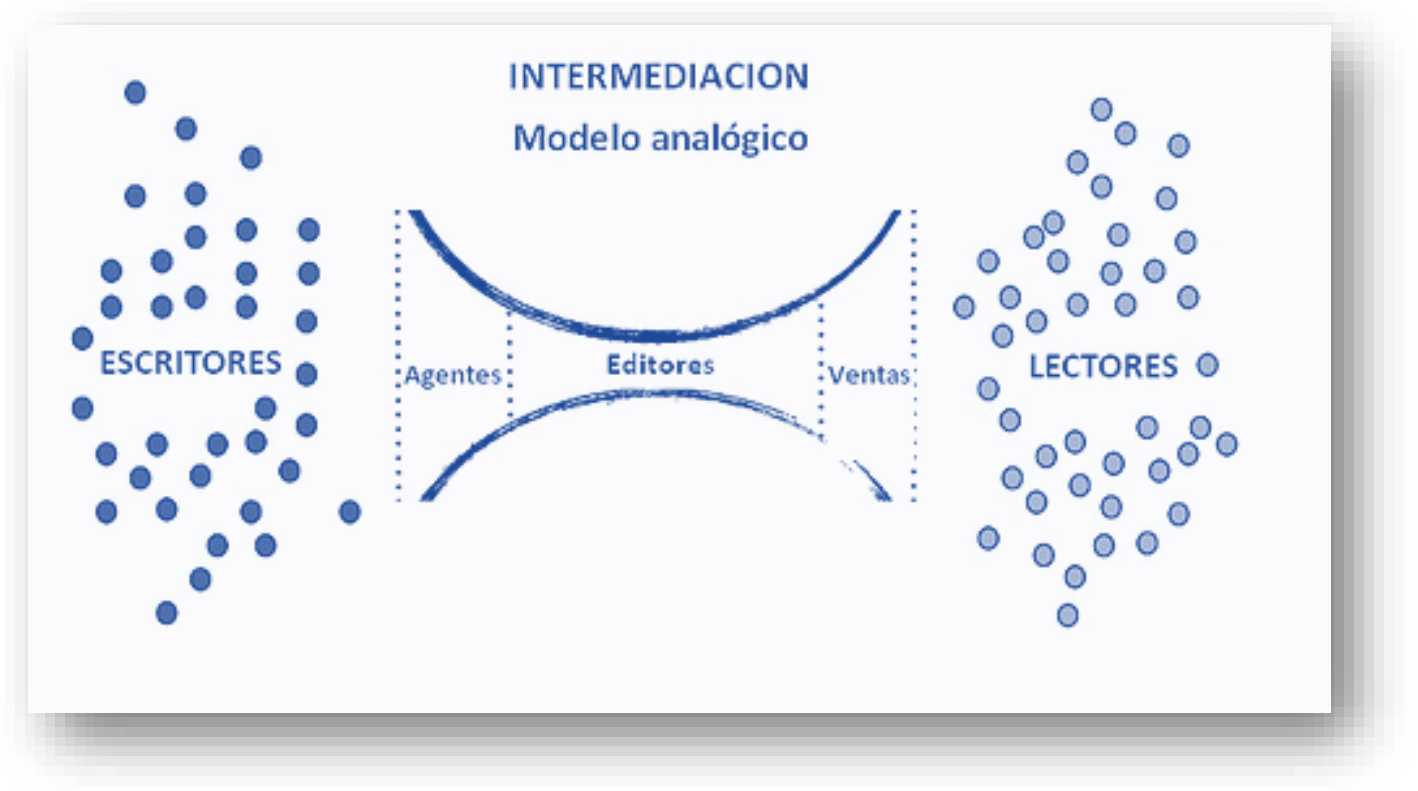

Otra característica común es que todos los agentes y representantes de autor que se están adaptando con éxito a las nuevas realidades editoriales lo están haciendo mediante la expansión de lo que significa ser un intermediario. Deshacerse de todos los intermediarios, no significa necesariamente que el autor no deje de ser un intermediario. Esto significa que tiene que especializarse, ser mejor. A medida que cambiamos de un paradigma de publicación de pocos a muchos a un entorno de muchos a muchos, el papel del agente no es sólo saber cómo adaptarse a los nuevos sistemas de publicación, lo que es relativamente fácil; más bien, se trata de utilizar todas las rutas posibles desde la concepción a la encarnación, desde el escritor al lector. 
Cada vez más, estas rutas se apoyan en las nuevas tecnologías, emplean nuevas estrategias de la comunidad, y se basan en modelos de utilidad que se resisten a ajustarse a cualquier estructura anterior. Sin embargo, tener conocimiento de esas rutas, y el equipo necesario para utilizarlas de manera efectiva, podría producir el tipo de valor tan necesario y beneficioso que han hecho históricamente los intermediarios.

Millones de escritores y millones de lectores no pueden encontrarse entre sí sin una introducción apropiada, por lo general organizada por un intermediario, ya sea su librero local o el motor de recomendación favorito del lector. Tradicionalmente los editores se han asociado a los autores para que les puedan comercializar sus obras los minoristas; los minoristas, recíprocamente, se han asociado a los lectores que están cerca de los editores. Pero, ¿qué han hecho los agentes? Tradicionalmente, los agentes han elegido autores para los editores: la búsqueda de autores, la segmentación poniéndoles en valor haciéndoles manejables y explotables para las masas.


INTERMEDIACION

Modelo digital

En la nueva economía editorial, los agentes necesitan tomar otra dirección. Los agentes como intermediarios del futuro son los agregadores de oportunidades, son quienes realizan la revisión de las plataformas, la segmentación, la definición de conocimientos, la evaluación y quienes aseguran la calidad. Una vez más los escritores tienen que ser conscientes de que necesitan un socio para publicar, no porque él o ella canalizaran 
los contenidos a través del estrecho canal de las grandes editoriales, pero si debido a que el agente, como una plataforma de publicación y un experto en su conexión con el lector, sabrá qué ruta tomar.

\section{La sociallización}

Entendemos por «lectura social» aquella que se desarrolla entornos virtuales en donde el libro y la lectura propicien la formación de una "comunidad» y alguna forma de intercambio. Por lo general se tiene como punto de encuentro una plataforma web o un software específico que organiza y proporciona a los usuarios un espacio de intercambio de información y de comunicación horizontal, donde las obras se evalúan y califican, además de compartir puntos de vista acerca de un texto, participando en grupos de discusión y/o elaborando por escrito comentarios y anotaciones sobre la obras y sus autores.

Teóricos como Chartier, McKenzie, Stein, etc. han estado discutiendo en los últimos años como la percepción cultural del libro como una unidad de totalización de la producción entra en contradicción con la heterogeneidad que implica la red, en la que la textualidad, carece de toda la carga simbólica del mismo. Esta disfunción opera a nivel del inconsciente colectivo en la aceptación de las nuevas formas de producción y reproducción de textos. Los debates, las discusiones y las actitudes a caballo entre lo apocalíptico y lo integrado responden a esta lógica. Los desafíos, cuando hablamos de lectura social, surgen desde el mismo objeto de estudio que no se restringe exclusivamente al ebook sino que afecta a todos los textos electrónicos.

Es el lector el que, en el tiempo, de acuerdo con los cambios que experimenta su contexto personal y el social, atribuye nuevos significados a obras que por su naturaleza textual inamovible permanecen estáticas en tanto que objetos. Los cambios de función de ciertas obras literarias, obedecen como es natural a las nuevas visiones del mundo aparecidas en la sociedad, en el ámbito lector. En este ámbito, y cuando se necesita o cuando se siente una carencia, surgen las nuevas lecturas, las nuevas comprensiones, las nuevas identificaciones. Como señala Mckenzie (2005), "Los nuevos lectores contribuyen a elaborar nuevos textos, y sus nuevos significados están en función de sus nuevas formas".

Decía Borges que toda lectura implica una colaboración y casi una complicidad, y así es. La historia de la escritura es la historia de la socialización de la misma; la invención de la imprenta incrementó exponencialmente, las audiencias e incluso el vocabulario disponible, de varios miles de palabras en el siglo $\mathrm{XV}$, a un millón en la actualidad; de manera similar la llegada del libro electrónico ha amplificado considerablemente la capacidad de lectura gracias a la naturaleza social de los contenidos que se imbrican en la cultura compartida de la filosofía de la web 2.0. Esta yuxtaposición de sus líneas fronterizas ha creado espacios novedosos y cambiantes en los que el concepto de libro se ha extendido a territorios antes 
inexplorados, lo que a su vez está repercutiendo en su visibilidad. Si a menudo se pone de manifiesto que la llegada de la digitalización está acabando con el libro y la lectura, las prácticas generales de lecto-escritura se están incrementando. Ahora más gente escribe, más gente lee, más autores publican a través de diferentes géneros como pueden ser blogs, prensa o redes sociales. Y al albur de todos estos cambios el mismo concepto de libro cambia (Alonso-Arévalo and Cordón-García 2014).

La lectura social responde a los modelos de aprendizaje 2.0, esto es: aprender haciendo, aprender interactuando, aprender buscando y aprender compartiendo. Las nuevas aplicaciones web simplifican tremendamente la cooperación y responden al principio de no requerir del usuario una alfabetización tecnológica avanzada para su utilización. Estas tecnologías de reciente masificación, estimulan la experimentación, generación y transferencia de conocimientos individuales y colectivos.

En el cambio de lo analógico a lo digital, el libro objeto desaparece y se convierte en una intefaz que se imbrica perfectamente con el software, en la que cobran importancia el acceso, lo abierto, la comunicación y lo social, conformando más como un sistema de comunicación que como un objeto. Los libros devienen en una experiencia compartida en un lugar en red, y la lectura que siempre fue social, se hace más SOCIAL. De este modo la «lectura social» se desarrolla en entornos virtuales en donde el libro y la lectura propician la formación de una «comunidad» y alguna forma de intercambio. Es un espacio de intercambio de información y de comunicación horizontal, donde las obras se evalúan, califican y etiquetan. Es habitual que los lectores compartan información sobre libros impresos y electrónicos que poseen, libros que han leído, libros que están leyendo, los que quisieran tener, y los que quisieran llegar a leer algún día. La llegada del libro electrónico ha amplificado considerablemente la capacidad de lectura gracias a la naturaleza social de los contenidos que se imbrican en la cultura compartida de la filosofía de la web 2.0. Esta yuxtaposición de sus líneas fronterizas ha creado espacios novedosos y cambiantes en los que el concepto de libro se ha extendido a territorios antes inexplorados, lo que a su vez está repercutiendo en su visibilidad.

El formato digital también abre otras posibilidades sociales que no se circunscriben exclusivamente a la lectura social, también la escritura social y la intervención de los lectores sobre el texto. Intervención que no es solo de la obra sobre el lector sino del lector sobre la obra en tanto colaborador en la elaboración de sentido configurando de este modo una suerte de palimsepto electrónico en el que tienen cabida todas las intervenciones de los lectores sobre la obra, de este modo aplicaciones como Openmargin o el club de lectura social de Amazon Kindle Cloud Reader permiten no sólo compartir un párrafo que hemos destacado, o una valoración sobre el libro, ya que además posibilitan conocer cuáles han sido los párrafos más destacados de 
ese libro, o ver todas las anotaciones que se hayan realizado sobre la obra. Si bien es cierto que este tipo de intervenciones se han hecho siempre sobre el libro, lo novedoso en que con el formato digital estás intervenciones pueden ser socializadas y compartidas de manera global.

\section{CONCLUSIÓN: EL NUEVO CONCEPTO DE LIBRO Y LAS NUEVAS FORMAS DE LECTURA}

Vivimos un momento de cambio, de transformación que nos retrotrae a los primeros momentos de la imprenta, cuando comienza a emerger un nuevo mundo y se va diluyendo el anterior, que se resiste a desaparecer. Vivimos en directo una revolución que, a diferencia de la de la imprenta, encierra en su seno decenas de revoluciones que se suceden casi a diario, microevoluciones de carácter técnico que van cambiando al mismo tiempo que los sistemas de lectura y escritura, la percepción y prácticas relacionadas con la misma. Vivimos instalados en un proceso de mutaciones permanentes en el que es difícil vislumbrar el futuro a largo plazo, pero en el que van surgiendo tendencias cada vez más consistentes que se van consolidando como factores inherentes al mismo.

Desde los años sesenta ya se había planteado la idea de que el libro impreso también era una tecnología «interactiva» e «hipertextual». Esto es particularmente cierto si pensamos que la «interactividad» del libro tradicional permite al lector acceder al texto desde múltiples niveles de interpretación y correlación de ideas, además de que el «soporte» es propicio para ser leído en fragmentos, estableciendo recorridos de lectura no lineales, en el que además se pueden introducir notas, huellas y marcas que -al igual que los hiperenlaces digitales - conducen el interés del lector hacia otros territorios y momentos de la narración, como es el pie de página. Los lectores van más allá de una mera reconstrucción narrativa en un orden predeterminado: deben desarrollar una actividad de construcción y reconstrucción de las secuencias para determinar y subsanar las deficiencias percibidas en el significado.

El libro y la lectura ha sufrido un cambio radical con la aparición de las nuevas tecnologías de la información, un cambio que incide en su mayor socialización y en su gestión compartida. Pero estos cambios están íntimamente ligados a la transformación que ha sufrido el libro a lo largo de los últimos años, en los hemos visto aparecer un nuevo tipo de paradigma vinculado igualmente con los cambios en sus formas de producción, reproducción y consumo. Estos cambios no afectan solamente a los procedimientos de lectura, sino a los intercambios sociales, a nuestras relaciones con el entorno y a todo el conjunto de prácticas relacionadas con el lenguaje (Cordón-Garcia et al. 2014). La necesidad de incorporar sustitutos funcionales a las prácticas desarrolladas en la cultura convencional obliga a la indagación, de forma sistemática, del entorno social, a la necesidad de adaptación al día a día de las innovaciones, a la conexión permanente a la red, que se ha convertido en el medio natural de los intercambios. 
Mientras los libros impresos han privilegiado la abstracción y la conceptualización, las nuevas tecnologías han permitido la configuración de nuevas identidades expresivas, donde el orden cognitivo, emocional y sociocultural, está siendo sustituido. Aún no sabemos de manera definitiva si esta nueva realidad es un cambio en positivo o en negativo en unos u otros aspectos. Muy frecuentemente nos encontramos con titulares de prensa que afirman una cosa o la contraria, que se lee más, que se lee menos en digital, que se lee más despacio, o más deprisa, que lo digital contribuye a una lectura no concentrada y dispersa, o bien que la percepción de la lectura no es sólo visual, y que las nuevas tecnologías favorecen un aprendizaje multimedia más completo; en la mayoría de estos casos los estudios cuentan con muestras muy fragmentadas y a veces apriorísticas de sólo unos pocos lectores analizados. Por ello debemos de ser prudentes, y esperar que haya estudios más extensos y contrastados.

Por lo que podemos decir que se está produciendo una asincronismo entre la progresión rápida de la tecnologías de la comunicación, por una parte, y la evolución de las prácticas de lectura de los usuarios por otra; originándose una asimilación lenta de estas por parte de las instituciones. Según estos expertos, nuestro cerebro no está aún capacitado para asumir el ritmo constante y la intensidad de estímulos cerebrales que comporta el consumo de cualquier tipo de contenidos culturales a través de los diferentes tipos de pantallas. Las nuevas generaciones se sienten muy cómodas con el uso de las herramientas 2.0, pero la mayoría desconocen los intereses comerciales, ideológicos y sociales que hay detrás de cada una de estas herramientas. La nueva percepción de la noción de lectura deriva de un entorno cultural en el que se produce un diálogo entre escritor y lector, cualidad que se magnifica en la red, donde ambos pueden interactuar en tiempo real, generando un nuevo paradigma de creación y recepción De cualquier forma, la participación activa o pasiva de los lectores en el uso de las nuevas tecnologías supone un proceso de readaptación profunda entre los seres humanos. La participación y el desarrollo de un conjunto de acciones desencadenadas por cualquier tipo de intervención nos sitúa ante un escenario nuevo, desde el punto de vista de la edición, de la lectura y de las formas comunicativas (Shirky 2012). Algunos autores llegan a hablar incluso de un proceso de mutación antropológica.

Si bien es preciso tener en cuenta el cambio natural de las generaciones de lectores, que se ven directamente influenciados por las mutaciones inducidas en cuanto a los dispositivos y las practicas de lectura. Y será esta primera generación que comienza a tener contacto desde la infancia con los dispositivos de pantalla táctil, tablet y smartphones, la que hará parte de su aprendizaje de lectura y de escritura sobre estos dispositivos digitales, y la que marcará sin ninguna duda la ruptura con el soporte impreso. Por lo que es preciso pensar la lectura más allá del libro, en un contexto de computación de lo real, con libros electrónicos que presentarán 
narratividades reticulares, no lineales, en un contexto aumentado de web en tiempo real, y de soportes de lectura que utilizarán nanotecnologías de almacenamiento. El futuro está por escribir.

Uno de los interrogantes que se planteaban en los comienzos de la contribución era el relativo a la incidencia de las nuevas tecnologías en las diferentes formas de lectura. Son muchas las voces que se han pronunciado al respecto, sobre todo desde el lado de los apocalípticos, en el sentido de subrayar los efectos de dispersión, falta de concentración, desmotivación, fragmentación, etc. imputables a la misma (Carr, 2011a, 2011b, 2014), o los perniciosos efectos fisiológicos de la misma (insomnio, problemas oculares, irritabilidad, desorientación), cuestiones que no dejan de ser anecdóticas frente a las evidencias de la progresión en lectura digital que se manifiesta en todos los países del mundo, sobre todo a partir de la implantación generalizada de los smartphones.

Más interesantes son los trabajos desarrollados por investigadores como Mangen $(2014,2015)$ en los que se analizan los procesos psicocognitivos implicados en el proceso de lectura a partir de la aparición de tecnologías y dispositivos de lectura nuevos y que intentan responder a interrogantes como $1 . \quad$ ¿Qué sabemos acerca de la forma en que interactuamos con los nuevos soportes que sustituyen a los medios convencionales? ¿Cómo se representa y gestiona el conocimiento y la experiencia del pasado a través de los nuevos medios? ¿Cuáles son las formas en que nos relacionamos con estos objetos de conocimiento y la información que contienen? ¿Qué impacto reviste la confluencia de formatos y medios en la experiencia de lectura? ¿Qué tipo de interacciones se producen entre los usuarios y los artefactos digitales?

De cualquier modo, la implicación y efectos de toda tecnología depende de todo un conjunto de procedimientos y competencias implicados en el proceso lector cuyo desarrollo puede verse mas o menos afectado por los desarrollos y nuevas propuestas tecnológicas.

Cada vez que se lee un texto se activan varias actividades

- Neuro-fisiológicas (movimientos oculares, funciones del cerebro, etc);

- Cognitivas

- Argumentativas / narrativas (el acto de seguir un mensaje complejo, como un discurso, una narración, etc);

- Afectivas (respuesta emocional)

- Simbólicas (interpretación del texto en el contexto de nuestro propio acerbo de conocimientos y establecimiento de las relaciones entre el texto que se lee y otros textos). 
Estos cinco procesos definen tres tareas: la manipulación (la dimensión material del proceso de lectura), la comprensión e interpretación. En otras palabras, al leer un texto se debe de ser capaz de progresar a través de él, lo que implica tanto aspectos manuales como neurofisiológicos. En una era post-tipográfica, debemos tener en cuenta cómo los cambios afectan a cada una de estas tareas y cómo las interrelaciones entre ellas se modifica también. ¿Qué significa manipular un libro electrónico? ¿Qué estrategias se deben desarrollar ahora que el elemento básico de la lectura, la página, no está presente? ¿Puede la manipulación trasladarse sin problemas de un contexto (la cultura del libro) a otro (la cultura de la pantalla o la cultura del libro electrónico)?

La segunda tarea, la comprensión, implica la dimensión semiótica del proceso de lectura. Leer un texto es entender lo que está escrito, lo que implica aspectos lingüísticos, cognitivos y afectivos. En los libros electrónicos se articulan nuevas textualidades, que requieren nuevas estrategias de comprensión.

La tercera tarea, la interpretación, se refiere a la dimensión simbólica del proceso de lectura. Leer es establecer una relación entre el texto que se lee y otros textos que explican, ilustran, completan o amplían lo que se lee. Si la interpretación es la relación establecida entre un mínimo de dos textos por un lector, un sistema de lectura en que se potencia la recuperación presumiblemente contribuirá a la mejora de la interpretación. Para ello es necesario estar en posesión de un conocimiento completo y complejo del texto que se lee, pues de lo contrario esa conexión puede confundir en lugar de facilitar la interpretación.

Las tres tareas implicadas, la lectura-interpretación, la comprensión, y la manipulación son revisten un carácter convergente. No pueden existir formas complejas de interpretación si no alcanza una forma adecuada de comprensión, lo que a su vez requiere de formas satisfactorias de manipulación.

Lo que está en juego ahora, con los libros electrónicos remita a esta primera fase, esto es, el dominio de las formas básicas de manipulación. Los nuevos libros requieren del desarrollo de estrategias formativas que faciliten la apropiación de dispositivos y aplicaciones, como paso previo a la optimización de la comprensión y de la interpretación.

Teniendo en cuenta todos estos factores cualquier definición de libro que se ensaye ha de contemplar los aspectos tecnológicos, contextuales e interpretativos, teniendo en cuenta el factor de credibilidad que juega en todo tipo de reconocimiento de géneros. Lejeune, se había referido al "pacto autobiográfico" para ilustrar el desplazamiento de la creencia del lector al autor cuando este afirma que lo que lee es su vida, el mismo tipo de fenómeno que se concita en una novela o en otro tipo de escrito. Esta suerte de acuerdo opera también 
cuando hablamos de definiciones. Un blog ¿es un libro? ¿Puede llegar a serlo? ¿Un conjunto de twitter? ¿Son un libro? ¿Pueden llegar a serlo? ¿Un audio ¿es un libro? ¿Puede llegar a serlo? Las blognovelas, la Twitliteratura, los audiolibros, constituyen respuestas positivas a estos planteamientos (Escandell, 2014). En su forma impreso el libro se definia, y se define, sobre todo por el continente, cualquier objeto con forma de volumen, que adquiere esa denominación y está dotado de un ISBN es considerado como tal por cualquier lector, independientemente de que su contenido sean un conjunto de entradas de un blog, una serie de Twitts o las entradas de un dietario, la forma y su presencia determina su contenido. Pero cuando hablamos de libro electrónico esta suspensión de la creencia es más difícil de depositar en un archivo o un conjunto de archivos textuales, iconográficos, sonoros, etc. donde las dimensiones tienen que ver no solo con la extensión en páginas, sino con la duración (audiolibros) y con las posibilidades de navegación e interrelación, a través de hipervínculos y redes sociales.

Por lo tanto cualquier definición de libro ha de instalarse en la provisionalidad, siempre dependiente de las innovaciones que se van pergeñando al hilo del tiempo, y en todo caso, como ocurre con el caso de la novela, una suerte de cajón de sastre que ha ido acogiendo todo tipo de variaciones, se puede decir, como en el manifiesto firmado por los gobiernos de Italia, Francia, Polonia y Alemania, para protestar por el diferente gravamen a los libros impresos y a los libros electrónicos (http://www.youscribe.com/catalogue/tous/education/declaration-conjointe-sur-la-tva-pour-le-livrenumerique-2560565) que un libro es un libro, independientemente de los soportes. 


\section{REFERENCIAS BIBLIOGRÁFICAS}

ABBA, TOM; BJARNASON, BALDUR (2015). This is not a book. http://thisisnotabook.baldurbjarnason.com/SinglePage.html

ALONSO-ARÉVALO, J. AND J. A. CORDÓN-GARCÍA 2014. Lectura Social, metadatos y visibilidad de la información. In Proceedings of the XLV Jornadas Mexicanas de Bibliotecología, Monterrey, Monterrey, México, 14-16 de mayo de 2014 2014, AMBAC ed. AMBAC. Disponible en Internet: <http://gredos.usal.es/jspui/handle/10366/123295>.

ASHLOCK, J. A. Redefining the Middleman. DBW, 2014, (January 10, 2014). Disponible en Internet: <http://www.digitalbookworld.com/2014/redefining-the-middleman/>.

BENHAMOU, FRANÇOIS (2014). Le Livre à l'heure numérique: Papier, écrans, vers un nouveau vagabondage. Seuil.

BURKE, PETER (2009). Los avatares de El Cortesano: lecturas y lectores de un texto clave del espiritu renacentista. Barcelona, Gedisa.

CARR, NICHOLAS (2011a). ¿Google nos hace estúpidos?. Madrid, Taurus

CARR, NICHOLAS (2011a). Superficiales: ¿qué está haciendo Internet con nuestras mentes? Madrid, Taurus

CARR, NICHOLAS (2014). Atrapados: cómo las máquinas se apoderan de nuestras vidas. Madrid, Taurus

CORDÓN GARCÍA, JOSÉ ANTONIO (2014). Participación, personalización e interacción: modelos y sistemas de lectura digital. En: Rossing, Tania; Rettemaier, Miguel (ed.). Leitura, arte e patrimônio: redesenhando redes. Universidad de Passo Fundo.

CORDÓN GARCÍA, JOSÉ ANTONIO; ALONSO ARÉVALO, JULIO; GÓMEZ DÍAZ, RAQUEL. Social Reading: Platforms, Aplications, Clouds and Tags. Oxford, Chandos Publishing, 2013. ISBN 9781 843347262. 
CORDÓN-GARCÍA, J.-A. AND J. ALONSO-ARÉVALO. Mediación y legitimación cultural: la impronta de las redes sociales. In Anuario ThinkEPI. EPI SCP, Barcelona, Spain, 2011, p. 264-268. Disponible en Internet: <http://eprints.rclis.org/16920/>.

CHARTIER, ROGER (2000). Las revoluciones de la cultura escrita. Barcelona, Gedisa.

CROUZET, THIERRY (2015). La mécanique du texte. Publie.net

CUDDYEN, G. Publishing's Digital Disruption Hasn't Even Started. DBW, 2015, (April 23, 2015).

Disponible en Internet: <http://www.digitalbookworld.com/2015/publishings-digitaldisruption-hasnt-even-started/>.

ESCANDELL, DANIEL (2014). Escrituras para el siglo XXI. Literatura y blogosfera. Madrid, Iberoamericana.

FURTADO, J. A. Self-publishing: ¿tsunami o "business as usual"? In Libros electrónicos y contenidos digitales en la sociedad del conocimiento: mercado, servicios y derechos. Madrid: Pirámide, 2013.

GENETTE, GERARD (2001). Umbrales, Barcelona, Siglo XXI.

KLEIN, NAOMÍ (2011). No logo: el poder de las marcas. Barcelona, Paidos.

MANGEN, A. \& KUIKEN, D. (2014) Lost in an iPad: Narrative engagement on paper and Tablet. Scientific Study on Literature, vol. 4, no 2, pp. 150-157

MANGEN, A., ANDA, L.G., OXBOROUGH, G. \& BRØNNICK, K. (2015) Handwriting versus typewriting: Effect on word recall. Journa of Writing Research

MCKENZIE, D. F. (2005). Bibliografía y sociología de los textos. Madrid, Akal.

PANOZ, JIMINY (2013). Design du livre numérique. Walruss

SCOLARI, CARLOS ALBERTO (2013). Narrativas transmedia: Cuando todos los medios cuentan. Deusto. 
SINOFSKY, S., B. PARTNER AND A. HOROWITZ The Four Stages of Disruption. recode.net, 2014, (January 6, 2014). Disponible en Internet: <http://recode.net/2014/01/06/the-four-stages-ofdisruption-2/>.

SOCCAVO, LORENZO (2015). Les mutations du livre et de la lectura, Uppr. 